\title{
L'ÉTUDE MATHÉMATIQUE DES ASSURANCES NON VIAGERES DANS L'EUROPE CONTINENTALE OCCIDENTALE
}

\author{
H. Ammeter, P. Depoid, B. De Finetti
}

Rapport à la Session d'ASTIN, Nere York, I957

\section{Avertissement}

Ce rapport n'a pas le but de résumer toute la littérature actuarielle sur les assurances non viagères: on se proposait de tracer le fil conducteur de quelques uns des développements d'idées où l'apport des mathématiques est plus profond et, en particulier, de ceux où il semble qu'il y aura encore à travailler longtemps. A ce compte, seulement quelques étapes pouvaient être explicitement mentionnées dans le texte, tandis qu'une bibliographie complète aurait dû suivre à la fin du rapport.

Maintes circonstances ont restreint le temps à disposition des rapporteurs et ont augmenté les difficultés d'un travail en commun à distance; sans doute quelques contributions ont échappé ou n'ont pas reçu le relief qu'il aurait fallu leur donner. Les auteurs prient de bien vouloir justifier les omissions par ces circonstances, et de les leur signaler.

\section{L'assurance en général et l'assurance non viagère}

L'assurance sur la vie et les assurances non viagères semblent présenter des différences essentielles, soit au point de vue de leur objet (indemnité d'une perte dans le deuxième cas, mais non dans le premier), soit au point de vue de leur méthode (théorie mathématique complétement développée dès longtemps pour le premier cas, mais non dans le deuxième).

Ya-t-il néanmoins une notion unique d'assurance, en général, qui comprenne toutes les deux? Est-il possible de concevoir d'une façon unique l'application du calcul des probabilités et des méthodes mathématiques qui le traduisent, dans le champ de l'assurance en général? Ou ya-t-il des limitations, ou des circonstances qui obligent à distinguer à cet effet les deux champs de l'assurance sur la vie et de l'assurance non viagère? 
Au point de vue juridique-économique, la conception unitaire s'est imposée surtout grâce aux ouvrages de U. Gobbi (3) et A. Manes (6), où la notion d'assurance est étendue jusqu'à comprendre toute opération ayant le but d'éliminer des risques pour quelqu'un.

Au point de vue mathématique, les doutes semblent s'apaiser seulement lors des Congrès int. d'actuaires de I937 (XI, Paris) et de I940 (XII, Lucerne) où ils étaient proposés à la discussion respectivement comme Thème VI (L'assurance incendie et l'assurance des choses) et Thème I (Le calcul des probabilités dans le domaine de l'assurance). Les aspects qui nous intéressent ont été discutés par les auteurs suivants des pays envisagés: (à la première occasion) B. de Finetti (I06) et L. Neumann (I22); (dans la deuxième) J. Baptist (I00), B. de Finetti (I08), W. G. J. ten Pas (I28), E. Göring (I09), I. Lah (II 8), et surtout dans la communication (III) et dans le rapport général (II2) de $\mathrm{H}$. Jecklin. A ce sujet, il convient de signaler aussi un autre exposé de ce dernier auteur (II3), de même que les études de W. Thalmann (I27) et de W. Wunderlin (129, 130).

Dans ce même ordre d'idées très général se placent les discussions sur les raisons qui rendent attrayante pour l'assuré une opération nécessairement défavorable (en tant qu'espérance mathématique): on trouve des explications qualitatives dans U. Gobbi (3), qui sont traduites d'après la notion d'utilité introduite dans "Theory of Games" par von Neumann et Morgenstern, p. ex. dans B. de Finetti (I07).

\section{Applications actuarielles et statistiques classiques}

Le mot "classique" est ici quelque peu excessif; on fait simplement allusion à l'ensemble des questions qui se trouvent exposées dans des traités bien connus et concernant des aspects actuariels et statistiques plutôt naturels. Il s'agit, au fond, de l'application du principe d'équivalence dans l'assurance des choses, en cherchant à déterminer et combiner les probabilités qui entrent en jeu par les voies les plus spontanées. Pour cette raison, et du fait qu'il s'agit surtout de travaux moins récents ou de nature didadique, nous nous limiterons à des indications sommaires.

Commençons par la France, où en I9o8 a paru l'ouvrage de $\mathrm{H}$. 
Goury (4), „Questions de technique et d'organisation pratique relatives aux assurances contre les accidents."

En I92I, P. Richard a publié la première édition en deux volumes de son traité: Théorie et pratique des opérations d'assurances, Une nouvelle édition, entièrement remaniée et étendue, a été publiée en I944-I946 (II). Le deuxième volume de cet ouvrage traite des assurances autres que l'assurance sur la Vie. Des développements importants sont consacrés aux assurances Maladie, R. C. Automobile, Incendie et autres dommages, Crédit, Accidents du travail et Réassurances.

L. Potin, après avoir publié en I934 (8) un ouvrage: Calcul des tarifs des assurances de risques divers, a publié en I948, sous le titre: Leçons sur la théorie mathématique des assurances accidents et de responsabilité civile (9), le texte du cours qu'il professait à l'Institut des Sciences Financières et d'Assurances de l'Université de Lyon. Cet ouvrage, très riche en considérations techniques, est divisé en 4 parties:

I. Calcul de la prime

2. Etude théorique d'un certain nombre de risques; R. C. Auto, Bris des Glaces, Bris des Machines, Grêle, R.C. Diverses, Accidents du Travail, Individuelle

3. Calcul des réserves - Réassurance - Surveillance des Risques 4. Incendie

Enfin, en 1950, P. Depoid (2) a publié sous le titre: Applications de la statistique aux assurances Accidents et Dommages, le texte de son cours à l'Institut de Statistique de l'Université de Paris. Ce volume renferme une abondante documentation sur les assurances Maladie, Individuelle Accidents, Accidents du Travail, R.C. Auto, Incendie et Grêle.

En Italie un traité par L. Molinaro (7) "Teoria e tecnica delle assicurazione elementari", contenant aussi des développements mathématiques, a paru en I946; en 1933 on y avait publié en italien le traité de N. Sergowskij ( $\mathrm{I} 6$ ) dont l'original allemand „Einführung in die Theorie der Feuerversicherung" (15) avait paru en I93I à Prague.

En Allemagne ont été publiés en I936 deux traités, l'un de P. Riebesell (I2), ,Einführung in die Sachversicherungsmathematik”, et l'autre de M. Gürtler (5), „Die Kalkulation der Versicherungs- 
betriebe", qui ont été mentionnés les derniers pour remarquer que ce sont les ouvrages qui (avec les autres publications de ces auteurs et de leurs élèves) ont peut-être contribué principalement à amener cette branche de la science actuarielle dans la direction des développements sur lesquels il faudra nous arrêter.

En dehors des traités, on aurait naturellement à mentionner un grand nombre de travaux publiés dans des revues ou présentés aux Congrès d'actuaires; on les trouvera dans la liste bibliographique, et - lorsqu'il y a une raison particulière — dans les exposés des prochains paragraphes.

\section{Expressions analytiques pour risques individuels}

L'échafaudage de notions donnant le fondement pour traiter mathématiquement les questions concernant les risques individuellement considérés, est très différent d'une branche à l'autre, d'après la nature des éventualités assurées.

Le cas de l'assurance maladie et le cas de l'assurance des accidents individuels (en particulier, accidents du travail et maladies professionelles), s'appuient sur les méthodes actuarielles employées en assurance sur la vie; en effet, tout élément du risque (probabilités, coût pour soins médicaux et indemnités journalières) est supposé fonction de l'âge, et, en se servant d'une table de survie $l_{x}$ on parvient à exprimer les primes pour opérations de longue durée par des procédés usuels tels que l'introduction de nombres de commutation convenables.

Sur ces sujets on peut signaler en particulier: pour la maladie, Walther $(5 \mathrm{I}, 52)$ Tosberg $(46,47)$ Galbrun (30) Dubourdieu (27); pour les accidents: Maingie (36), Zwinggi (55) Carus (2I).

Des expressions analytiques ont été proposées dans ces champs pour des questions ultérieures qu'il peut y avoir intérêt à analyser: en particulier les classifications d'après cause et durée de la maladie, surtout avec rapport aux limitations de durée de la couverture. Ainsi M. A. Coppini (25) trouve que la distribution des cas de maladie d'après la durée $u$ peut s'exprimer comme combinaison linéaire de deux distributions du type respectif $u^{p} e^{-a u}$ et $u^{q} e^{-b / u}$ (caractéristiques pour maladies de nature courte et longue). Sur le cas de longue maladie voir aussi R. Risser (4I) et H. Burckhardt (I9). 
D'une façon analogue K. Böschenstein (x8) a traité ces problèmes en introduisant les fonctions de Bessel.

La plupart des autres assurances non viagères exigent d'analyser la prime à fixer d'après des éléments logiquement plus simples dont elle dépend: essentiellement, la probabilité de sinistres ${ }^{1}$ ) et la distribution de probabilité des sinistres d'après le montant du dommage. L'une et l'autre dépendent d'une foule de circonstances variables; doit-on viser à individualiser les risques ou à les grouper en négligeant les différences individuelles? et comment dresser et utiliser les statistiques? faut-il recourir à ces méthodes d'augmentation ou réduction de la prime standard (en mesure absolue ou en pourcentage) pour tenir compte de circonstances défavorables ou favorables? nous ne nous arrêtons pas sur ces aspects, que l'on peut trouver p. ex. dans Molinaro (I87).

Ce qui se rattache mieux au sujet de cet exposé, c'est plutôt la possibilité de relier des notions et des grandeurs à d'autres. Un exemple intéressant est la distinction de Riebesell (I2) d'une "probabilité d'embrasement" (Ausbruchswahrscheinlichkeit) et d'une "probabilité de propagation" (Ausbreitungswahrscheinlichkeit): la probabilité d'incendie pour un bâtiment se compose de la probabilité d'embrasement (incendie y ayant origine) et de celles d'embrasement des bâtiments voisins combinées avec les probabilités respectives de propagation.

En ce qui concerne la distribution de probabilité du sinistre d'après le montant du dommage, il y a lieu de remarquer la différence entre le cas où une limite supérieure existe (destruction totale d'un objet assuré) ou n'existe pas (responsabilité: il peut arriver de próvoquer des dommages aussi grands que l'on veut). Indiquons (pour plus de simplicité, avec référence à ce dernier cas) la distribution par $s(z)$ (Schadentafel, d'après Gürtler): dans le cas de couverture totale, l'indemnité moyenne est évidemment

$$
\bar{s}=\int_{0}^{\infty} z s(z) d z
$$

1) En général, on considère les probabilités annuelles; normalement elles sont petites et alors ce fait n'introduit pas d'erreur appréciable. Mais il ne faut pas oublier qu'il serait exact de transposer les raisonnements usuels aux intensités $\mu(\mu d t=$ probabilité dans un temps $d t)$, et non sur les probabilités $q=\mathrm{I}-e-\mu=\mu-\frac{\mu^{2}}{2 !}+\frac{\mu^{3}}{3 !} \cdots$ 
et la prime $P=q \bar{s}$ ( $q=$ probabilité de sinistre). L'écart quadratique de l'opération peut se calculer de la même façon: il est donné par $\sigma^{2}=q(1-q) \bar{s}^{2}+q \bar{\sigma}^{2}$ (le premier terme est celui d'une assurance ou pari avec enjeu $\bar{s}$ sûr ; le deuxième dépend du caractère aléatoire de l'indemnité, $\sigma$ étant l'écart quadratique moyen de la distribution $s(z)$. Il suffit de développer

$$
\sigma^{2}=(\mathrm{I}-q)(\mathrm{o}-q \bar{s})^{2}+q \int_{0}^{\infty}(z-q \bar{s})^{2} \mathrm{~s}(z) d z
$$

en posant $(z-q \bar{s})=(z-\bar{s})-(\mathrm{I}-q) \bar{s}$

Connaître, en plus de $\bar{s}$, la distribution $s(z)$ n'est cependant pas superflue; au contraire cela est nécessaire afin de déterminer la prime dans les cas de franchise (intégrale ou à déduire), de limitation de couverture (comme premier risque), etc. Sur ces mêmes bases, on peut étudier - comme l'ont fait Riebesell et quelques-uns de ses élèves - l'incidence des mesures préventives sur le montant des primes, et en déterminer l'optimum (minimum de dépenses).

Nous n'avons rappelé ces relations préliminaires que pour mentionner, à l'égard de l'allure de $s(z)$, quelques expressions analytiques proposées en raison de leur simplicité ou de leur accord avec des matérieaux statistiques particuliers.

La plus simple est l'exponentielle: $s(z)=k e^{-c z}$ (Riebesell, I4); ces autres types simples de fonctions de fréquence analytiques ont été indiquées par M. Gürtler (5)
A) $s(z)=4(\mathrm{I}-z)^{3}$, (prédominance de petits dommages)
B) $s(z)=3 / 2-6(z-\mathrm{r} / 2)^{2}$ (pr. de dommages moyens)
C) $s(z)=4 z^{3}$
D) $s(z)=\mathrm{I} 2(\mathrm{I} / 2-z)^{2} \quad$ (grands et petits, peu de moyens),

la fonction

$$
s(z)=k \frac{I}{z} e^{-(a \log c z)^{2}}
$$

(distribution de McAlister, connue particulièrement d'après les applications de M. Gibrat) a été proposée par L. Amoroso (6I) et R. d'Addario (57).

Profitons de l'occasion pour mentionner une autre expression analytique donnée par les mêmes auteurs sur un sujet qui ne trouve sa place logique ni ici ni ailleurs dans cet exposé: elle concerne les 
sinistres en réserve, c'est-à dire non encore réglés, après un laps de temps donné. $(58,59,62)$.

4. Modèles explicatifts des phénomènes globaux (cas d'indépendance)

En supposant que les phénomènes élémentaires que l'on envisage soient indépendants (pour mieux spécifier: stochastiquement indépendants), les conclusions concernant les phénomènes globaux qu'ils déterminent seraient bien simples. Si compliquées que puissent être les lois des faits élémentaires, le théorème "central" du calcul des probabilités nous assure que pour les grandeurs globales (sous des restrictions triviales par rapport à nos applications) la distribution normale (de Gauss) s'applique nécessairement. Comme tout le monde le sait, elle a la densité

$$
f(x)=k e^{-\frac{1}{2} \frac{(x-m) 2}{\sigma^{2}}}
$$

avec $m=$ espérance mathématique, $\sigma=$ écart quadratique moyen ( $k=1 / \sqrt{2 \pi} \sigma$ n'est que le facteur de normalisation).

Si le nombre aléatoire $x$ est le nombre de sinistres (en général: de réalisation parmi un groupe d'évènements indépendants), avec probabilité $q$ égale pour tous, ladite distribution apparait comme forme limite de la distribution binômiale pour laquelle ( $x=$ entier, $f(x)$ probabilité de la valeur $x$ et non densité)

$$
f(x)=\left(\begin{array}{l}
n \\
x
\end{array}\right) q^{x}(\mathrm{I}-q)^{n-x}
$$

lorsque $n \rightarrow \infty$, pourvu de prendre $m=n q, \sigma=\sqrt{n q(\mathrm{I}-q)}$. Mais la même conclusion demeure valable si les probabilités $q_{1} \ldots q_{n}$ sont différentes (alors $m=\Sigma q_{i}, \sigma=\sqrt{\Sigma q_{i}\left(\mathrm{I}-q_{i}\right)}$ ), pourvu toujours qu'il y ait indépendance stochastique.

Le même passage à la limite, si on imagine la probabilité $q$ très petite (ou les $q_{1} \ldots q_{n}$ très petites), ou - mathématiquement - si l'on tient fixe $m=n q$ (ou $m=\Sigma q_{i}$ ), conduit à l'autre distribution bien connue sous le nom de distribution de Poisson; la probabilité de la valeur $x$ ( $x$ entier!) est

$$
f(x)=m^{x} e^{-m / x !}
$$

( $m$, comme ci-dessus, est l'espérance mathématique; dans ces exem- 
ples, en particulier, $m=n q$ ou $m=\Sigma q_{i}$; $\sigma$ est ici nécessairement $\sqrt{m})$.

Ces conclusions s'étendent aisément au cas où le nombre aléatoire auquel on s'intéresse n'est plus le nombre $x$ des sinistres, mais le total $z$ des indemnités relatives. Si le nombre des sinistres est grand, $z$ obéit à la distribution normale (avec $m=$ espérance mathématique des sinistres $=$ prime pure totale, et $\sigma^{2}=\Sigma \sigma_{i}^{2}, \sigma_{i}$ $=$ écart quadratique moyen de chaque assurance

$$
\sigma_{i}{ }^{2}=q_{i}\left(\mathrm{I}-q_{i}\right) \bar{s}_{i}{ }^{2}+q_{i} \bar{\sigma}_{i}{ }^{2}
$$

Lorsque le nombre des sinistres $x$ n'est pas si grand, et que pour $x$ il faut de ce fait appliquer la distribution de Poisson, la distribution pour $z$ est une généralisation de celle de Poisson donnée par la densité

$$
f(z)=\sum_{0}^{\infty} \frac{m^{h}}{h !} e^{-m} s^{* h}(z),
$$

ou $s^{* h}(z)$ est la puissance de plissement (Faltung) d'ordre $h$ de $s(z)$ concernant chaque sinistre. L'application de cette distribution de Poisson généralisée (introduite par de Finetti et Khintchine dans l'étude des processus stochastiques homogènes discontinus) dans les problèmes des assurances de choses est due à Ackermann (I32) et développée par plusieurs auteurs: Anft (I4I), Campagne-de JonghSmit (I44), F. P. Cantelli (I49), etc.

\section{Même sujet (sans restriction d'indépendance)}

Comme on a eu le soin de la placer constamment sous les yeux, l'hypothèse d'indépendance stochastique constitue une restriction tellement extrème et tellement simplificatrice, que les formules du $\mathrm{n}^{0} 4$ - formules quelque peu compliquées pour traiter d'un cas presque trivial — ont leur intérêt, plus qu'en soi, comme introduction aux extensions où l'on essaye de tenir dûment compte des interdépendances. Si l'indépendance est une hypothèse très simple, la non indépendance est quelque chose de largement indéterminé (c'est dire: après avoir considéré les droites, voyons les lignes qui ne le sont pas). Il y a beaucoup de méthodes que l'on peut essayer d'appliquer, beaucoup de schémas théoriques capables de fournir 
des explications convenables, sur la voie à suivre pour nous affranchir de la restriction de l'indépendance.

Ce n'est pas par ailleurs un désir de généralité des mathématiciens qui soulèvent cette question; il a été remarqué maintes fois que les écarts dans les fréquences des décès, des incendies, etc. sont plus grands que dans des jeux de hasard et c'était facile de le prévoir, en considérant les épidémies, les influences du climat, etc.

Etant donné que l'interdépendance concerne surtout les événements, on peut supposer encore indépendents les montants des différentes indemnités, et appliquer toujours la formule (I) sauf laisser indeterminés les coefficients $w_{h}$ (probabilité de $h$ sinistres) au lieu de supposer comme jadis $w_{h}=m^{h} e^{-m / h}$ ! d'après la formule de Poisson. On aura ainsi

$$
f(z)=\sum_{0}^{\infty} w_{h} s^{* h}(z)
$$

Il reste à choisir des modèles (plus ou moins persuasifs en soi) qui portent à déterminer les $w_{h}$ (de façon à obtenir $f(z)$ suffisamment en accord avec les données d'observation). Voici quelques-unes de ces méthodes (qui comme on va le voir, sont étroitement liées entre elles).

En imaginant que l'interdépendance est due à une sorte de contagion, et en appliquant alors le schéma d'urnes élargi étudié par G. Polya et F. Eggenberger (ro3), on est conduit a l'expression

$$
w_{h}=m(m+d)(m+2 d) \ldots(m+(h-\mathrm{I}) d) / h !\left[(\mathrm{I}+d)^{1 / d}\right] m+h d
$$

(où, encore, $m=n q$, et $d$ est un "paramètre de contagion"); si $d=0$, on retombe dans le cas de Poisson.

On peut imaginer que les événements sont échangeables ${ }^{1}$ ), ce qui revient au même que de supposer que la probabilité $q$ est choisie au hasard (une foi pour toute l'année) avec une certaine densité $\mathrm{Q}(\xi)$ (c. à. d.: $Q(\xi) d \xi$ est la probabilité de choisir $q$ entre $\xi$ et $\xi+d \xi$ ). L'expression de $w_{h}$ est donnée alors par la correspondante combinaison de distributions poissonniennes:

"Ou "équivalents" (de Finetti (IO5)) ou "symétriques" (Savage (I 25)); la dénomination "échangeables" (Fréchet) paraît préférable étant la seule qui n'admet pas - semble-t-il - d'ambiguité d'interprétation. 


$$
w_{h}=\frac{\mathrm{I}}{h !} \int_{0}^{1}(n \xi)^{h} e^{-n \xi} Q(\xi) d \xi
$$

Cette méthode a été appliquée par H. Ammeter (I33), qui a en particulier choisi comme distribution $Q(\xi)$ une du type Gamma, en démontrant que dans ce cas on trouve le même résultat que dans le cas de contagion.

Une autre formule permet de déterminer les $w_{h}$ en fonction d'autres valeurs $\tau_{1}, \tau_{2}, \ldots \tau_{k}$.. ayant la signification de valeurs moyennes du nombre de sinistres simples, doubles, ..., multiples d'ordre $k, \ldots$ dans un schéma donné par $\mathrm{H}$. Pollaczek-Geiringer (I23) et discuté par H. Lüders (II9). L'expression, quelque peu compliquée, est:

$$
\omega_{n}=e^{-\left(\tau_{1}+\tau_{2}+\ldots+\tau_{n}+\tau_{n+1}+\ldots\right)} \sum \frac{\tau_{1}^{r_{1}} \tau_{2}^{r_{2}} \ldots \tau_{n}^{\gamma_{n}}}{r_{1} ! r_{2} ! \ldots r_{n} !}
$$

la sommation s'étendant sur toutes les combinaisons

$$
\left.r_{1}+r_{2}+r_{3}+\ldots r_{n}=n\right) .
$$

Ce cas se ramène encore à celui de Polya-Eggenberger si l'on pose en particulier

$$
\tau_{j}=k \frac{\mathrm{I}}{j}\left(\frac{d}{\mathrm{I}+d}\right)^{j} \quad(k=m / d),
$$

(et, évidemment, à la distribution de Poisson si en plus $d=0$, c.à.d. si $\tau_{1}=m, \tau_{j}=0$ pour tout $j>\mathrm{I}$, ou en somme s'il n'y a pas de sinistres multiples).

Ce type de formulation devrait être utile dans des cas tels que les accidents auto, où les $\tau_{i}$ pourraient résulter d'hypothèses théoriques en fonction de l'intensité de la circulation, ou de statistiques des accidents de circulation (Lüders (II $)$ )).

La connaissance exacte des distributions n'est pas cependant nécessaịre dans la plupart des cas; si on veut utiliser quelques moments de $z$ seulement, on peut les exprimer (avec référence au schéma présent) par les moments de $x$ (c. à. d.: de la distribution $w_{h}$ ) et ceux de chaque sinistre (c.à.d.: de la $s(z)$ ). Voir Böhm (I42), et J. Janko (I7I). 
M. Hofmann (IIO) a étudié l'applicabilité de processus composés de Poisson à plusieurs dimensions dans l'assurance accidents. B. H. de Jongh (II4) a traité un système de risques interdépendants où les événements secondaires, tertiaires etc. provenant du même événement primaire ont été groupés en générations. A cet effet, il s'est servi du schéma des processus stochastiques des naissances (pure birth process).

On peut naturellement se passer du schéma (2), et aborder directement l'étude de la distribution du total des sinistres $z$, ou de quelques moments de $z$. Pour ce qui est de l'écart quadratique moyen, il suffit tout simplement de considérer les coefficients de corrélation, $r_{i j}$, entre chaque couple de risques; il est bien connu en effet que, si $z=\sum z_{i}$ ( $z_{i}$ sinistre pour chaque assurance), on a

$$
\sigma(z)=\sqrt{\Sigma_{i} \sigma^{2}\left(z_{i}\right)+2 \sum_{i j}^{*} r_{i j} \sigma\left(z_{i}\right) \sigma\left(z_{j}\right)}=\sqrt{\overline{\Sigma \bar{\sigma}_{i}{ }^{2}+2 \Sigma_{i j}^{*} r_{i j} \bar{\sigma}_{i} \bar{\sigma}_{j}}}
$$

(ou le* signifie somme étendue aux couples $i, j$ avec $i<j$ ). Noter que si, en particulier, on suppose $\bar{\sigma}_{i}=\bar{\sigma}=$ const., et $\mathrm{r}_{i j}=\mathrm{r}=$ const. (comme nécessairement dans l'hypothèse d'équivalence d'Ammeter), on a tout simplement.

$$
\sigma(z)=\bar{\sigma} \sqrt{n+r n(n-\mathrm{I})}=n \bar{\sigma} \sqrt{r} \sqrt{\mathrm{I}+\frac{\mathrm{I}-r}{r n}} \approx n \bar{\sigma} \sqrt{r}
$$

Il est bon de remarquer que la validité de la loi des grands nombres (même dans l'interprétation la plus faible, de tendance vers zéro de l'écart quadratique moyen relatif) est strictement conditionnée par l'absence d'une corrélation positive: s'il y a une corrélation positive $(r>0)$ si petite que ce soit, l'ordre de grandeur de $\sigma(z)$ croît toujours comme $n$ (non comme $\sqrt{n}$ ) et $\bar{\sigma}(z) / n$ tend vers une constante positive (vers $\bar{\sigma} \sqrt{\gamma}$ ) et non vers zero ${ }^{\mathbf{1}}$ ).

Et on peut enfin, en dehors de tout schéma théorique et de tout essai interprétatif, envisager brutalement le problème d'adapter aux données statistiques des types convenables de distributions avec le seul but de simplification descriptive. A cet effet on emploie le plus souvent les courbes de Pearson; Riebesell et ses élèves, surtout C. Lange (II7) (voir aussi L. Neumann (I22)), ont procédé à de telles interpolations. Ces interpolations, quelque peu mécaniques, ne

1) Rappelons qu'une corrélation négative ne peut subsister systématiquement: la formule (3), en effet, donnerait un résultat absurde. 
astisfont toutefois guère à la longue, car les résultats dépendent constamment des observations et expériences faites, et leur application à d'autres catégories d'assurances reste problématique.

\section{Les fluctuations périodiques: cas de l'assurance grêle}

Dans le cas de fluctuations présentant une certaine régularité de périodicité, le problème est tout à fait différent du cas des fluctuations aléatoires, accidentelles, irrégulières. Le cas des fluctuations périodiques semble effectivement se produire pour l'assurance grêle, et il semble précisément confirmé que la période coïncide avec celle des tâches solaires.

Cela avait été constaté en I93I par A. Piron (9I) (expérience de quelques compagnies françaises); en I934 V. Myslivec (90) a confirmé ces conclusions, en appliquant des méthodes plus adéquates (méthode d'autocorrélation, d'après Fuhrich (87)), et en utilisant des expériences de plusieurs pays. Voir aussi l'étude critique par H. Jecklin (88) de toute la littérature se rattachant à cette question.

On peut remarquer encore que l'existence de ces fluctuations a conduit à des discussions sur l'opportunité d'envisager une compensation des risques dans le temps outre que dans l'espace; voir, p. ex. F. J. Weiss (94).

\section{Théorie du risque}

Dans l'assurance de choses - bien plus que dans l'assurance sur la vie - la compensation du risque et les mesures nécessaires à son maintien constituent deux facteurs d'une importance fondamentale. Il n'est donc pas surprenant qu'une grande partie de la littérature sur l'assurance de choses se rapporte aux problèmes de la théorie du risque.

Dans la théorie du risque, il y a lieu de distinguer les critères suivis d'après deux points de vue. Le premier concerne l'étendue de temps auquel on étend les prévisions: dans la théorie dite classique il s'agit d'une période limitée (un exercice, un petit nombre d'exercices, la durée d'extinction d'un portefeuille existant); dans la théorie dite collective, on envisage des règles de conduite valables pour toujours. L'autre point de vue coincide avec la distinction posée entre les cas du n.4 et n.5: avec ou sans restriction d'indé- 
pendance (distinction qui s'applique par ailleurs seulement si on exprime le risque global comme composé par les risques individuels).

La théorie classique du risque est très ancienne, mais s'occupait systématiquement de l'assurance vie. Les premiers résultats ayant intérêt pour n'importe quelle branche sont peut-être ceux de Cantelli (I49) et Medolaghi (I83/4), concernant des inégalités du type de celle de Bienaymé. Pour ce qui est de l'affranchissement de l'hypothèse d'indépendance, le premier à introduire les corrélations (selon la ligne suivie ici au n.5, formule (3)) a été P. Dubois (I54). Il faut noter que, à la rigueur, la théorie collective suppose elle-même l'hypothèse d'indépendance (ce serait un handicap très lourd, ne fût-il possible de l'enlever).

Plusieurs travaux (p.ex.ceux mentionnés à propos de la distribution de Poisson généralisée, n.5, formule (2)) s'attachent à des questions sur les réserves de risque et les chargements de sécurité. Ces méthodes sont plus perfectionnées que les précédentes basées sur la mesure (quelque peu grossière mais toujours fondamentale du risque) donnée par l'écart quadratique moyen, en tant qu'elles introduisent des distributions (ou, du moins, des moments d'ordre supérieur etc.); toutefois, lorsqu'elles négligent l'interdépendance (comme dans le cas dont nous parlons), on est amené à juger qu'il était plus substantiel de considérer (même quelque peu grossièrement) l'effet éclatant de la corrélation que de préciser la forme d'une distribution de signification fictive, faute de tenir compte de cela.

La théorie collective du risque, créée depuislongtempspar Lundberg (I79) (dont l'exposé plus complet est en suédois (I80)), a attiré l'attention de la généralité des actuaires après l'exposé de $\mathrm{H}$. Cramèr (I5I). Il s'agit d'établir des règles pour l'attribution de bénéfices à un fond de sécurité, de façon que la probabilité de faillite demeure trés petite même dans une durée tendant à l'infini. L'analogie avec le problème célèbre de la ruine des joueurs est manifeste; de Finetti (I62, I64) l'a développée en montrant que les conclusions mêmes de la théorie collective du risque se rattachent directement aux méthodes et résultats classiques de De Moivre (qui, de plus, en mettent en lumière la vraie raison d'être).

Le sujet a été développé et enrichi par J. Dubourdieu (I57); A. Thépaut (209) en a fait le fondement d'applications pratiques; et il 
est intéressant d'ajouter que M. Massé (I82) et d'autres économistes français ont fait application de ce principe dans la théorie des décisions économiques dans les situations comportant du risque.

Ammeter (I33) a généralisé la formule (2) pour le cas de fluctuations des probabilités de sinistres et a étudié une série d'applications de cette théorie au domaine de l'assurance de choses, par exemple en déterminant les limites de l'assurabilité (I34). Dans le rapport (138) figure une bibliographie détaillée concernant la théorie collective du risque.

Latscha ( $\mathrm{r} 75)$ a examiné au moyen de la théorie collective du risque quelques aspects de l'assurance sociale contre les accidents. Citons en outre le mémoire de Campagne et Driebergen (I47) qui ont publié des études fondamentales sur le critère de solvabilité dans l'assurance de choses en faisant appel à la notion de la probabilité de ruine.

Les modèles issus de la théorie collective du risque, susceptibles d'être encore développés, constituent indubitablement une base appropriée et dynamique pour l'évolution de la science actuarielle dans l'assurance de choses, fondée sur la théorie des probabilités (Ammeter I34).

Cependant, des doutes sur le fondement du critère de base de la théorie collective ont été soulevés; entre autres considérations, $\mathrm{G}$. Ottaviani (rgI), B. Tedeschi $(203,4,5)$ C. Campagne (I45) et C. Campagne et C. Driebergen (I47) ont remarqué le fait indiscutablement étrange, que le critère à suivre afin de limiter la probabilité de ruine doit être de plus en plus sévère. Ce fait a été examiné aussi par Dubourdieu et Thépaut (dans les travaux que l'on vient de mentionner); l'expédient qu'ils suggèrent semble cependant incompatible avec les prémisses. Ces réflexions ont amené de Finetti (I6I) à se rallier aux critiques exposées au dessus et à chercher une modification effective de la théorie collective, apte à enlever la difficulté. Le développement de cette idée a fait l'objet d'une communication (I64) presentée au XV me Congrès.

\section{Problèmes de la réassurance}

Les problèmes de réassurance touchant le domaine de l'assurance des choses sont en rapport étroit avec les questions de la théorie du risque soulevées au n.7. En effet, le but de la réassurance est de diminuer le risque; il s'agît d'établir de quelle façon ce but peut 
être atteint plus efficacement avec le moindre sacrifice, ou en réalisant les objectifs accessoires de simplicité administrative; il y a alors à approfondir les problèmes particuliers de chaque forme de réassurance; il reste enfin à examiner l'effet d'ensemble de la répartition des risques dans un réseau de réassurance.

Le problème classique consiste dans la détermination. du plein, en supposant les cessions effectuées par excédent; on peut distinguer la question des pleins relatifs (de façon à conserver des quote-parts des différents risques en proportions cohérentes) et celui des pleins absolus (de façon à atteindre un degré de sécurité convenable).

Plusieurs travaux s'occupent du plein relatif; entre autres G. Tolentino (2II) (résultats de nature générale, bien qu'appliqués au cas vie), B. de Finetti (I59) (critère: maximiser la réduction de risque pour une perte de profits fixée), G. Ottaviani ${ }^{1}$ ) (I95).

Le problème du plein absolu a été examiné au point de vue de la théorie du risque classique et de celle collective; au même ordre d'idées se rattache la question des limites d'assurabilité et des risques catastrophiques. Voir H. Ammeter (I40), J. Sousselier (199), et presque tous les travaux cités pour le plein relatif. Voir aussi Lasheras-Sanz (I74), Wyss $\left(\mathbf{2} \mathbf{I}_{3}, 4\right)$ et J. Loisel (I78) qui s'applique à un cas spécial, etc.

La réassurance en excédent comporte cependant de lourds frais administratifs; on a imaginé des procédés pratiques pour réduire les travaux, comme celui, très ingénieux, introduit par G. Destradi et décrit dans ( $\mathrm{I}_{53}$ ); plus radicalement, on est passé à des systèmes tout à fait nouveaux, comme l'"Excess of Loss" et le "Stop Loss".

$\mathrm{K}$. Klinger ( $\mathrm{I} 72$ ) a étudié un type spécial de contrat "Stop Loss" prévoyant des franchises différentes pour chaque classe de dommages partiels.

H. Ammeter (I37), en se fondant sur la théorie collective, a exposéle problème du plein et celui de la tarification de ces dernières formes de réassurance. Pour calculer la prime nette de la réassurance „Stop Loss", on pourra se servir par exemple de la formule

$$
P^{L}=\int_{k P}^{\infty}(x-k P) d F(x, n q)
$$

1) Ici c'est Giovanni Ottaviani, frère de Guiseppe Ottaviani, Auteur de tous les autres travaux portant ce nom. 
$\mathrm{kP}$ représentant le plein de l'assureur cédant et $\mathrm{F}(\mathrm{x}, n q)$ résultant de la formule (I).

Le chargement de sécurité devant être ajouté à la prime nette est calculé en fonction de l'écart quadratique des prestations excédant la valeur présumée ou selon la probabilité de ruine relative à la réserve de sécurité accumulée par le réassureur. La méthode, utilisée pour la tarification dans la réassurance "Stop Loss" s'applique non seulement à la réassurance proprement dite, mais elle fournit aussi un procédé permettant de déterminer les bénéfices sur le risque; dans ce cas, les primes "Stop Loss" sont considérées comme réserve interne pour les risques non encore courus (I37).

Sur les mêmes thèmes un exposé très complet est dû à J. Sousselier et M. Ramel (200); Mlle. G. Braicovich (I43) a discuté quelques points; parmi les nombreuses variantes des nouvelles formes de réassurance mentionnons l'ECOMOR proposé par A. Thépaut (208).

Enfin, lorsqu'on passe l'examen des effets d'ensemble engendrés par le marché de la réassurance, on parvient à trouver rationnelle (d'après $B$. de Finetti (I6o, I6I) une réassurance générale de quote parts (comme si tous les assureurs s'unissaient dans un pool mondial); G. Ottaviani (I92) a perfectionné cette analyse en tenant compte d'autres facteurs; B. de Finetti (I63) a examiné l'effet des superpositions de couvertures d'un même risque parvenant à un réassureur de différentes voies, et en particulier le cas où cela a lieu par des traités de "Stop Loss".

\section{Conclusion}

En conclusion, nous croyons pouvoir dire que la longue énumération des travaux effectués montre l'intérêt que les actuaires de l'Europe continetale occidentale ont, depuis longtemps, porté aux assurances des risques non viagers.

En I948, l'écossais J. G. Kyd publiait dans le B.A.R.A.B. un article dans lequel il exprimait le souhait que soit élargie la sphère d'influence de l'actuaire, l'expérience du passé montre que, depuis longtempś, l'actuaire a cessé de se cantonner dans l'assurance vie. Il n'en reste pas moins que de nombreuses tâches restent à entreprendre et qu'un large domaine est encore ouvert à ses investigations. 


\section{LISTE BIBLIOGRAPHIQUE}

\section{LES MÉMOIRES DÉSIGNÉS PAR UN * CONTIENNENT D'AUTRES RENSEIGNEMENTS BIBLIOGRAPHIQUES}

\section{ABRÉVIATIONS:}

BIAF Bulletin de l'Institut des Actuaires Français, Paris.

BISFA Bulletin de l'Association des Actuaires diplômés de l'Institut des Sciences Financières et d'Assurances de l'Université de Lyon.

BARAB Bulletin de l'Association Royale des Actuaires Belges, Bruxelles. BAAS Bulletin de l'Association des Actuaires Suisses, Berne.

BANAI Bolletino del l'Associazione Nazionale degli Attuari Italiani, Roma. AINA Atti dell'Istituto Nazionale delle Assicurazioni, Roma.

GIIA Giornale dell'Istituto Italiano degli Attuari, Roma.

PSS I problemi del servizio sociale, Roma.

BVM Blätter für Versicherungsmathematik oder Blätter der Deutschen Gesellschaft für Versicherungsmathematik, Berlin/Köln.

VAAB Verzekerings-Archief, Actuarieel Bijvoegsel, 's-Gravenhage.

WR Wirtschaft und Recht der Versicherung, Berlin.

SVJ Statistische Vierteljahresschrift, Wien.

CR CIA Comptes-rendus d'un Congrès International d'Actuaires.

\section{A. OUVRAGES GENERAUX}

[I] Burrau, C.: Die Grundlagen der Versicherungsstatistik, WR 1924.

[2] Depord, P.: Applications de la statistique aux assurances accidents et dommages, Paris I950.

[3] GоввI, U.: L'assicurazione in generale, Padoue 1938.

[4] Goury, H.: Questions de technique et d'organisation pratique relatives aux assurances contre les accidents, Paris 1908.

[5]* Gürtler, M.: Die Kalkulation der Versicherungsbetriebe, Berlin I934.

[6]* Manes, A.: Versicherungswesen, 5. Auflage, Leipzig 1932.

[7] Molinaro, L.: Teoria e tecnica delle assicurazioni elementari, Rome I946.

[8] Potin, L.: Calcul des tarifs des assurances de risques divers, Paris I934.

[9] Leçons sur la théorie mathématique des assurances accidents et de responsabilité civile, Lyon 1948.

[Io] Poznanski, T.: Réflexions sur l'assurance des dommages, CR XI CIA, Paris I937.

[rI]* Richard, P.: Théorie et pratique des opérations d'assurances, nouvelle édition, Paris 1944-46.

[12]* Riebesell, P.: Einführung in die Sachversicherungsmathematik, Berlin 1936.

[13] Fragen aus der Feuer- und Sachversicherung, CR XI CIA, Paris 1937.

[I4] Die mathematischen Grundlagen der Sachversicherung, CR XII CIA, Lucerne I940. 
[I5] SergowskiJ, N.: Einführung in die Theorie der Feuerversicherung, Prague I93r.

[I6] Introduction à la theorie de l'assurance incendie, Turin I933.

\section{B. ASSURANCES MALADIE ET ACCIDENTS}

[I7] BERGER, J.: Über die Deckungsrückstellung der vertraglichen Krankenversicherung, Deutscher Aktuarverein I95I.

[18] Böschenstein, K.: Der Reduktionsfaktor in der Theorie der Krankenversicherung und die Besselschen Funktionen, BAAS 1907.

[I9]* BuRckhard, H.: Beitrag zu einer Erweiterung der Krankenversicherung auf Ausgesteuerte, Berne I939.

[20] BühlmanN, $\mathrm{H}$. und HartmanN, W.: Änderungen in der Grundgesamtheit der Betriebsunfallkosten, BAAS 1956.

[2I] Carus, E.: Mathematische Grundlagen der Unfallversicherung mit Prämienrückgewähr, BVM I93I.

[22] Clamagirand, H.: L'etude de l'assurance des risques de maladies d'origine professionelle, BIAF I922.

[23] Coppini, M. A.: Prima indagine sul costo dell'assistenza sanitaria in relazione al sesso ed all'età degli assistiti, PSS I950.

[24] Nuove indagini sul costo dell'assistenza sanitaria in relazione al sesso ed all'età degli assistití, PSS I954.

[25] Sui fattori di riduzione e sulla distribuzione delle malattie per durata, PSS 1956.

[26] Dick, W.: Die Entkrankungsfläche, Bienne I947.

[27] Dubourdieu, G.: Les principes fondamentaux du calcul des probabilités et la théorie de l'assurance maladie, Paris 1939.

[28] FinetTI, B. DE: Il beneficio „operazioni chirurgiche”, CR X CIA, Rome I934.

[29] Gaillard, E.: Diverses études relatives aux accidents du travail, BIAF I902, I903, I904, I906, I9I0.

[30] Galbrun, H.: Théorie mathématique de l'assurance maladie, Paris I934.

[3I] Goury, H.: Assurance maladie, BIAF 1936.

[32] KIEnast, A.: Über die Berechnung des Reduktionsfaktors in der Krankenversicherung, BAAS 1923.

[33] Klinken, J. VAN: Statistical methods to inquire if the risk of accidents has changed, VAAB 1957.

[34] LePREUX, O.: La réparation des accidents du travail, BARAB Igo5.

[35] Lomnicki, Z.: Quelques observations au sujet de l'influence de l'âge sur la fréquence des accidents et les charges de réparation, CR XI CIA, Paris I937.

[36] MaIngie, L.: L'assurance contre les accidents du travail, BARAB I905.

[37] Ortaviani, Gius: Sul coefficiente di morbilità, PSS 1950.

[38] Razous, P.: La morbidité et la mortalité des professions dangereuses, BIAF I904.

[39] Rapport présenté sur le problème de l'établissement des statistiques ..., CR XI CIA, Paris I937.

[40] Risser, R.: Problème de l'établissement de statistiques ..., CR XI CIA, Paris 1937. 
[4I] A propos de l'assurance longue maladie, BIAF I956.

[42]* Robert, J.-P.: Bases techniques des assurances en cas d'hospitalisation, BAAS I953.

[43] Rusam, F.: Grundzüge der Mathematik der privaten Krankheitskostenversicherung, CR XII CIA, Lucerne I940.

[44] Spring, O. W.: Beiträge zur Theorie der Krankenversicherung, Berne I934.

[45] TARZE: Ies assurances contre les accidents du travail en France, Paris 1907.

[46] Tosberg, A.: Rechnungsgrundlagen und Schadentafeln der Krankheitskostenversicherung, Berlin I940.

[47] Über ein neues versicherungsmathematisches Verfahren als Ergebnis neuerer Morbiditätsuntersuchungen, BVM I953.

[48] Valencia, J. L.: L'assurance maladie, quelques idées sur la technique de l'assurance ..., CR IX CIA, Stockholm I930.

[49] VyDRA, V.: La technique de l'assurance maladie en Tchéchoslovaquie, CR IX CIA, Stockholm I930.

[50] WAGNER, H.: Zur technischen Behandlung der privaten Krankenversicherung, CR IX CIA, Stockholm I930.

[5I] WaLther, F.: Eine Morbiditätstafel für die Krankenpflegeversicherung, BAAS I938.

[52] Neue Morbiditätstafeln für die Krankengeldversicherung, Berne I945.

[53] WEBER, L. : Les accidents du travail en France, CR II CIA, Londres 1898.

[54] Wunderlin, W.: L'influence de l'âge sur la fréquence des accidents et le coût des prestations d'assurance, CR XI CIA, Paris 1937.

[55] ZwINGGI, E.: Mathematische Grundlagen der Unfallversicherung, BVM I93I.

\section{ASSURANCES RESPONSABILITÉ CIVILE ETC.}

[56] Abate, M.: Il rischio infortuni e le assicurazioni di responsabilità civile in rapporto alla circolazione stradale, Atti $2^{\circ}$ Congr. Naz. Scienza Assic., Trieste 1932.

[57] D'ADDario, R.: Curve di frequenza nelle assicurazioni di infortuni e di responsabilità civile, Rivista italiana di statistica, economia e finanza, Rome 1933.

[58] - Il calcolo della riserva-sinistri nelle assicurazioni elementari, GIIA I933.

[59] Considerazioni intorno alla riserva-sinistre Assicurazioni I934.

[60] Osservazioni sulla tariffa nelle assicurazioni danni, CR XI CIA, Paris 1937.

[6I] Amoroso, L.: Curve di frequenza nelle assicurazioni di infortuni e di responsabilità civile, Le Assicurazioni d'Italia 1932.

[62] La rappresentazione analitica delle curve di frequenza nei sinistri di infortuni .., CR X CIA, Rome I934.

[63] BURLot, A.: Etude de la fréquence des sinistres au tomobile, BIAF I936.

[64] Chossat, M.: Fréquence et probabilité en assurance accidents, Moniteur des Assurances 1937.

[65] DepoId, P.: Essai de synthèse des travaux statistiques relatifs à l'assurance RC automobile. CR XIII CIA, Scheveningen I95I. 
[66] Goury, H.: Assurance automobile, BIAF 1936.

[67] Henry, M. : Etude sur le coût moyen des sinistres en RC automobile, BIAF 1937 et 1938.

[68] Pellegrin, P.: Tarification de l'assurance auto, BIAF I948.

[69] Richard, P.: Les assurances de responsabilité civile - statistiques et tarifs - ,Paris I9I4.

[70] Risser, R.: A propos de quelques applications ... à l'étude de l'assurance accidents, BIAF 1936.

[71] De la fréquence des sinistres dans l'assurance auto, Revue générale des Assurances terrestres I939.

\section{ASSURANCE INCENDIE}

[72] Barrots, Th.: Essai sur l'application du calcul des probabilités aux assurances contre l'incendie, Mémoires Soc. Royale des Sciences, Lille I834.

[73]. Blandin, A.: Les bases techniques de l'assurance contre l'incendie, Paris I956.

[74] Bernus, P.: Sur une méthode rationnelle du calcul des primes d'assurance incendie, CR XI CIA, Paris 1937.

[75] Cantelli, F. P.: I fondamenti matematici della tecnica delle assicurazioni, GIIA I942.

[76] Le variabili casuali nell'assicurazione incendi, Soc. Italiana di Statistica r945.

[77] Coetnic, L.: Risque mathématique dans l'assurance contre l'incendie des immeubles, CR IX CIA, Stockholm I930.

[78] Courlleaux, L.: Réflexions sur l'assurance contre l'incendie, BIAF 1925.

[79] Depord, P.: Le rôle de la statistique en assurance incendie, Bull. SGF I94I.

[80] Duchez, E.: Etude sur l'organisation d'un service statistique centralisé en matière d'assurances incendie des risques industrielles, BIAF I948.

[8I] Neumans, L.: Zusammenhang zwischen Wirtschaftsablauf und Brandschadenverlauf, Schweizerische Versicherungs-Zeitschrift I954.

[82] Probleme aus der Feuerversicherung, Linz I955.

[83]. Razous, P.: L'assurance incendie des bois et forêts, BIAF I927 et I930.

[84] Soulier, P.: Note sur une théorie mathématique des assurances contre l'incendie, BIAF I 892 .

[85] Takidani, Z. et Shirasugi, S.: Theorie de Tarifstatistik in der Feuerversicherung, CR XI CIA, Paris I937.

\section{E. ASSURANCE GRELE}

[86] Beaucaron, J. R. DE: L'assurance contre la grêle en France et à l'etranger, Paris 1937.

[87] FUhRICH, J.: Über die numerische Ermittlung von Periodizitäten und ihre Beziehung zum Zufallsgesetz, Prague I933.

[88]* Jecklin, H.: Die Problematik der Hagelvoraussage, AssekuranzJahrbuch 1943 . 
[89] Medolaghi, P.: Sui fondamenti matematici dell'assicurazione contro i danni della grandine, AINA 1938.

[90] Myslivec, V.: Beitrag zur Theorie der Hagelversicherung, BVM 1936.

[9I] Piron, A.: Les orages à grêle en France, Paris I93I.

[92] Raillard, G.: En marge de la réserve de sécurité en assurance grêle, CR XI CIA, Paris 1937.

[93] Rossmann, G.: Ajustement des écarts en assurance grêle, BIAF 1938.

[94] WeIss, F. J.: Statistische Probleme der Hagelversicherung, CR XI CIA, Paris I937.

\section{F. BRANCHES DIVERSES}

[95] Alting von Geusau, G.: Etude statistique et technique du risque d'aviation, CR XI CIA, Paris I937.

[96] Henry, M.: Rapport sur l'assurance-aviation en France, CR XI CIA, Paris 1937.

[97] Hrlgard, E.: Die Gefahr des Luftfahrtwesens im Hinblick auf die Personen- und Sachversicherung, CR XI CIA, Paris I937.

[98] MedolaGHi, P.: La tecnica dell'assicurazione contro la disocupazione, AINA 1934.

[99] Tariffe variabili nelle assicurazioni danni, Soc. Italiana di Statistica I943.

\section{G. THEORIE DES PROBABILITÉS}

[IOo] Baptist, J.: Le calcul des probabilités dans le domaine de l'assurance, CR XII CIA, Lucerne I940.

[IoI] Consael, R.: Sur les processus de Poisson du type composé, Ac. R. de Belgique, Mém, Cl. Sci. I952.

[IO2] Dubourdieu, F.: Remarques relatives à la théorie mathématique de l'assurance accidents, BIAF I938.

[IO3] EgGENBERGER, F.: Die Wahrscheinlichkeitsansteckung, BAAS I924.

[I04] FinetTr, B. DE: Le funzioni caratteristiche di legge istantanea dotate di valori eccezionali, „Rend. Lincei”, Vol. XIV, I93I.

[I05] Classi di numeri aleatori equivalenti, ,Rend. Lincei", Vol. XVIII, I933.

[I06] Riflessioni teoriche sulle assicurazioni elementari, CR XI CIA, Paris 1937.

[IO7] Ai margini del dominio della matematica nei problemi dell'assicurazione, Assicurazioni 1938.

[108] Il calcolo delle probabilità nel dominio dell'assicurazione, CR XII CIA, Lucerne I940.

[I09] GöRING, E.: Eine Erweiterung der Mises'schen Kollektive und der entsprechende Ausbau der Theorie der Wahrscheinlichkeitsrechnung, CR XII CIA, Lucerne I940.

[IIo]* Hofmann, M.: Über zusammengesetzte Poisson-Prozesse und ihre Anwendung in der Unfallversicherung, BAAS I955.

[III]* JeckLIN, H.: Ist die Anwendbarkeit der Wahrscheinlichkeitstheorie in der Lebensversicherung besser fundiert als in der Sachversicherung ? CR XII CIA, Lucerne I940.

[II2] Die Wahrscheinlichkeitstheorie im Versicherungswesen (Rapport général), CR XII CIA, Lucerne I940. 
[II3] Die Wahrscheinlichkeitstheorie im Versicherungswesen, BAAS I94I.

[II4] JONGH, B. H. DE: Ein System von gegenseitig stochastisch abhängigen Risiken, VAAB I957.

[II5] Khintchine, 'A.: Asymptotische Gesetze der Wahrscheinlichkeitsrechnung, Erg. Math. 2, H. 4, I933.

[ir6] KolodziejCzYK, St.: Sur les événements à probabilité élevée CR XII CIA, Lucerne I940.

[II7] LANGE, C.: Untersuchungen über die jährlichen Schwankungen der Schadenquotienten in der Lebensversicherung und in der Feuerversicherung, WR I932.

[II8] LAH, I.: Wahrscheinlichkeitsrechnung und Versicherungswesen, CR XII CIA, Lucerne 1940.

[II9] LÜDERS, H.: Die Statistik der seltenen Ereignisse, Biometrika Ig28.

[I 20] Über die Verteilung der Schadenquotienten bei Hagelversicherungsunternehmungen, Neumanns Zeitschrift I934.

[I2 I] Masciotti, R. : La méthode actuarielle dans le domaine du continu, CR XIII CIA, Scheveningen I95I.

[I 22] Neumann, L.: Ủber die Anwendbarkeit der mathematischen Theorie in der Feuer- bezw. Sachversicherung, CR XI CIA, Paris 1937.

[123] Pollaczek-Geiringer, H.: Über die Poissonsche Verteilung und die Entwicklung willkürlicher Verteilungen, Zeitschrift für angewandte Mathematik und Mechanik I928.

[124]* Polya, G.: Sur quelques points de la théorie des probabilités, Ann. Inst. Henri Poincaré 1930.

[125] Savage L. T.: The foundations of statistics, New York I954.

[126] SergowskiJ, N.: Schwankungsintensität der Schadensquote und Schwankungsreserve in der Feuerversicherung, CR XI CIA, Paris 1937.

[127] Thalmann, W.: Die Frage der Aufstellung von Weltstatistiken über Arbeiterunfälle ..., CR XI CIA, Paris I937.

[I28] TEN PAS, W. G. I.: Wahrscheinlichkeitsrechnung und Statistik in der Versicherungsmathematik, CR XII CIA, Lucerne, I940.

[129] WUNDERLIN, W.: Anwendbarkeit der Wahrscheinlichkeitstheorie in der Unfallversicherung, BAAS I936.

[130] Zur Frage der Anwendung der Wahrscheinlichkeitstheorie in der sozialen Unfallversicherung, Lucerne 1942.

[I3 $\left.{ }^{2}\right]$ YNTEMA, L. : Einiges zur Wahrscheinlichkeitsansteckung, VAAB I954.

H. THÉORIE DU RISQUE, RÉASSURANCE ETC.

[I32] AckermanN, W. G.: Eine Erweiterung des Poissonschen Grenzwertsatzes und ihre Anwendung auf Risikoprobleme der Sachversicherung, Berlin 1939.

[133]* Ammeter, H.: Die Elemente der kollektiven Risikotheorie von festen und zufallsartig schwankenden Grundwahrscheinlichkeiten, BAAS I949.

[I34] La théorie collective du risque et l'assurance de choses, BAAS I954.

[r35] Risikotheoretische Methoden in der Rückversicherung, CR XIV CIA, Madrid I954.

[136] Über die risikotheoretischen Grenzen der Versicherbarkeit, BVM 1955. 
Voir aussi les ouvrages publiés dans les CR XIV CIA, Madrid I954 (sujet I).

[137] The calculation of premium rates for excess of loss and stop loss reinsurance treaties, Non proportional reinsurance, Bruxelles 1955.

[138] Literaturbericht über die kollektive Risikotheorie, BVM 1956.

[r39] Die Ermittlung der Risikogewinne im Versicherungswesen auf risikotheoretischer Grundlage, BAAS 1957.

[I40] Anwendungen der kollektiven Risikotheorie auf Probleme der Risikopolitik in der Sachversicherung, CR XV CIA, New York 1957.

[I4I] ANFT, K.: Utber die Bestimmung der Schwankungsreserve in der Feuer- und Hagelversicherung, Jena 1938.

[I42]* BoEHM, C.: Versuch einer systematischen Darstellung der modernen Risikotheorie, BVM I936.

[I43] BRAICovich, G.: Le riassicurazioni di danni nel quadro del problema riassicurativo generale, Non proportional reinsurance, Bruxelles 1955 .

[I44] Campagne, C., Jongh, G. H. DE, en Smit, J. N.: Bijdrage tot de wiskundige theorie van de bedrijfsreserve en het eigenbehoud in de brandverzekering, La Haye I947.

[I45] Campagne, C.: L'assurance contre les dommages et la théorie du risque collectif, VAAB 1955 .

[ $\left.\mathrm{r}_{4} 6\right]$ La théorie mathématique de la réserve technique dans l'assurance incendie, Annales de Sciences Actuarielles, Louvain 1956.

[147] Campagne, C. und Driebergen, C.: Das Solvabilitätskriterium in der Schadenversicherung, VAAB 1957.

[I 48] The influence of chain reactions in the loss distribution function, CR XV ClA, New York 1957.

[r49] Cantelli, F. P.: Intorno a un problema fondamentale della teoria del rischio, BANAI I9II.

[150] Su due applicazioni di un teorema di G. Boole alle statistica matematica, Rome I9I7.

[I5I]* Cramér, H.: On the mathematical theory of risk, Stockholm 1930.

[I52] Collective risk theory, Stockholm I955.

[I53] Destradi, G. ${ }^{1}$ : Simplification of surplus treaty administration, Quarterly letter, Alg. Herverz. Mij., Amsterdam I952.

[I54] Dubors, P.: Essai d'application du calcul des probabilités aux problèmes concernant la réassurance, BIAF 1936.

[I55] Contributions a l'étude de la réassurance, CR XI CIA, Paris 1937.

[I56] A propos d'un essai de réassurance en excédent de sinistres en Norvège, BIAF 1938.

[I57] Dubourdieu, J.: Théorie mathématique du risque dans les assurances de répartition, Paris 1952.

[I58]* FinetTI, B. DE: La teoria del rischio e il problema della rovina dei giocatori, GIIA 1939.

[159] Il problema dei pieni, GIIA I940.

[I60] Einzelwirtschaftlicher und gemeinwirtschaftlicher Gesichtspunkt in der Frage der Rückversicherung, BVM I942.

[I6I] Impostazione individuale e impostazione collettiva del problema della riassicurazione, GIIA I942.

1 Exposé anonyme tiré des circulaires de M. Destradi aux réassureurs des Ass. Gen. Trieste, après le décès de l'auteur. 
[162] La compensazione tra rischi eterogenei, GIIA r954.

[I63] L'intreccio riassicurativo, CR XIV CIA, Madrid I954.

[I64] Su un' impostazione alternativa della teoria collettiva del rischio, CR XV CIA, New York I957.

[I65] FrancoIs, J. L.: Essai d'ajustement d'une distribution de sinistres, BISFA I95I.

[I66] Franckx, E.: Contribution à l'étude des variables aléatoires composées, CR XIV CIA, Madrid I954.

[167] Borne du risque dans l'assurance élémentaire, CR XV CIA, New York 1957 .

[I68] FÜRST, D.: La rovina dei giocatori nel caso di riserva limitata, GIIA 1956.

[i69] Hagstroem, M.: La loi de Pareto et la réassurance, Stockholm I925.

[I 7o] Hochart, M.: Essai d'une théorie du plein, BIAF I923.

[I7I] Janko, J.: Les méthodes de réassurance en Tchécoslovaquie, CR XI CIA, Paris I937.

[I72] KLINGER, K.: Über eine Form des Rückversicherungsvertrages zur Erreichung normaler Schwankung des Schadensatzes, Berlin 1939.

[I73] LANDRÉ, C. L.: Aperçu succinct des théories du plein de l'assurance, CR II CIA, Londres I 898.

[I74] Lasheras-Sanz, A.: Apportation à l'établissement d'une théorie mathématique de la réassurance, CR XIV CIA, Madrid I954.

[I75] Latscha, R.: Zur Anwendung der kollektiven Risikotheorie in der schweizerischen obligatorischen Unfallversicherung, BAAS 1956.

[I76] Leepis, P.: Über die Anwendbarkeit von Durchschnittsverfahren zur Bestimmung der Schadenreserve in der privaten Unfallversicherung, BAAS I944.

[177] LefìvRe, J.: Application de la théorie collective du risque à la réassurance excess-loss, Skandinavisk Aktuarietidskrift 1952.

[178] LoIsel, J.: Essai de tarification rationnelle de la réassurance en excédent du risque de non-payement des loyers, CR XI CIA, Paris 1937.

[I79] LundberG, F.: Zur Theorie der Rückversicherung, CR VI CIA, Vienne rgog.

[180] Försäkringsteknisk riskutjämning, Stockholm I926-28.

[I8I] Mangiapan, J.: Sinistres et risques en assurance, BISFA 1956.

[182] Massé, P.: Les réserves et la régulation de l'avenir dans la vie économique, Paris 1946.

[183] Medolaghi, P.: Di una nuova teoria del rischio, BANAI igo8.

[184] La teoria del rischio e le sue applicazioni, CR VI CIA, Vienne rgog.

[185] Il rischio nelle assicurazioni, AINA I930.

[I86] Indirizzi di ricerca nell'assicurazione danni, GIIA I938.

[187] Molinaro, L.: La tecnica della riassicurazione nei rami elementari, Assicurazioni I95I.

[188] NeumanN, L.: Der Ertragsquotient in der Rückversicherung, Mitteilungen der Vereinigung kantonalschweizerischer Feuerversicherungsanstalten I953.

[I89] Risikotheorie und Feuer-Überschadenrückversicherung, Die Versicherungsrundschau I954.

[I9o] Niklaus, Th.: Réflexions sur une théorie générale des assurances, CR III CIA, Paris Igoo. 
[I9I] Ottaviani, Gius.: La teoria del rischio del Lundberg e il suo legame con la teoria classica del rischio, GIIA I942.

[192] Sul problema della riassicurazione, GIIA I952.

[I93] Sulla riassicurazione, CR XIV CIA, Madrid I954.

[I94] Alcune considerazioni sulla teoria collettiva del rischio, CR XV CIA, New York I957.

[195] Otraviani, Giov.: Considerazioni sul problema del pieno relativo nell'assicurazione incendi, GIIA I95I.

[I96] QUINKERT, W.: Die kollektive Risikotheorie unter Berücksichtigung schwankender Grundwahrscheinlichkeiten mit endlichem Schwankungsbereich, Karlsruhe 1957.

[197] RICHARD, P.: Sur un nouveau mode de réassurance, BIAF I949.

[r98] Schmetterer, W.: Die Risikotheorie in der Versicherungsmathematik, SVJ 1956.

[I99] SousseliER, J.: L'assurance des risques catastrophiques, BISFA I953.

[200] Sousselier, J. et Ramel, M.: De la determination et de l'indétermination des primes d'excédents de sinistres, Non proportional reinsurance, Bruxelles I955.

[20I] Steindler, A.: Sulla determinazione numerica della funzione di ripartizione della distribuzione di Bravais, Trieste 1956.

[202] TAUBER, A.: Bedenken gegen das Aequivalenzprinzip in der Risikotheorie, Versicherungsarchiv 1934.

[203] Tedeschi, B.: Alcune considerazioni sulla teoria classica e sulla teoria collettiva del rischio, GIIA 1951.

[204] Ulteriori considerazioni sulla teoria classica del rischio e altri esempi relativi alle varie teorie, Rome I95I.

[205] Concezioni teoriche del rischio e loro aderenza alla realtà delle assicurazioni, Atti IV Congr. Un. Mat. Ital., Taormina I95r.

[206] Thépaut, A.: Sur la détermination du bénéfice industriel dans les compagnies d'assurances contre les accidents, Paris I933.

[207] Sur la réserve pour sinistres restant à régler, BIAF I935.

[208] Le traité d'excédent du coût moyen relatif ECOMOR, BIAF I950.

[209] Essai de détermination pratique des pleins de conservation, BIAF I953.

[210] Calcul du plein de conservation dans l'assurance des corps de navire, CR XV CIA, New York I957.

[2II]. Tolentino, G. : Sul pieno di conservazione nell'assicurazione vita, Atti $2^{\circ}$ Congr. Naz. Scienza Ass. Trieste 1932.

[212] WolfF, K. H.: Ein Beitrag zur kollektiven Risikotheorie, SVJ 1956.

[213] Wyss, H.: Die Risikotheorie und ihre Bedeutung für die Versicherungsmathematik, BAAS 1953.

[2I4] Die Risikotheorie als Grundlage für die versicherungsmathematische Behandlung von Rückversicherungsproblemen, CR XIV CIA, Madrid 1954. 